\title{
Improvement of Survivability of a Power Plant's Direct Current Auxiliary Power System under Electromagnetic Pulse (HEMP)
}

\author{
Vladimir Gurevich, Ph.D. \\ Central Electrical Laboratory Israel Electric Corp. Haifa, Israel \\ *Corresponding Author: Vladimir Gurevich, Central Electrical Laboratory Israel Electric Corp. Haifa, \\ Israel

\begin{abstract}
High Altitude Electromagnetic Pulse (HEMP) is a challenging agent aimed to destroy the most critical types of electronic equipment in the most important components of national infrastructure. The article addresses the issue of improvement of a power plant's direct current auxiliary power system's (DCAPS) survivability under HEMP impact, and suggests certain simple measures and affordable technical means that ensure significant improvement of DCAPS's resistance to HEMP.
\end{abstract}

Keywords: Power Plant, Electromagnetic Pulse, HEMP, Direct Current Auxiliary Power System

\section{INTRODUCTION}

HEMPs, as well as special high-power sources of pulse electromagnetic emission, are the most up-todate and effective means to destroy the electronics of power systems, communications, water supply systems and other critical parts of national infrastructure facilities, without direct damage to the population [1,2]. Thus, this kind of weapon is deemed to be the most promising and is in rapid evolution in all developed countries.

A direct current auxiliary power system (DCAPS) represents an important power plant's system, the lack of which makes it impossible for other systems to operate. A DCAPS of each power plant's block usually consists of two powerful high-capacity accumulator batteries, two powerful battery chargers (BC), DC switchboards (or DC cabinets). The DC distribution system is very branchy and contains many long non-shielded cables spread over a vast territory. In case of HEMP impact, this cable system will act as a huge antenna that absorbs the energy from the vast area and brings it directly to very sensitive digital systems of the power plant's management, control, protection, signaling and communication systems. However, those are not the only devices that need to be protected from HEMP. The DCAPS itself, with its electronic BC, also requires protection. Modern $\mathrm{BC}$ are equipped with micro-electronic and more often a microprocessor-based system of control that is very sensitive to HEMP. Thus, they need to be protected in the first turn.

\section{Improvement of Protecting LeVel of A BC Cabinet}

Powerful BC, intended for currents of several hundreds of Amps, are designed as metal cabinets consisting of 2-3 compartments that are usually located close to each other. Figure 1 shows three BC for $300 \mathrm{~A}$ (one serves as a backup). Each of them is designed as a 2-compartment metal cabinet. A well-designed metal cabinet provides good protection to internal electronic equipment from HEMP. Do usual cabinets of usual BCs feature the same qualities? Definitely, they don't! Figure 1 shows "weaknesses" of these cabinets. These include: open top (1) intended to improve ventilation and cooling; the panel of digital programming and measuring unit (2); ventilation grid at the bottom of the cabinet's door (3) and the open bottom of the cabinet (4). In addition to that, attention should be paid to the rubber seals running along the doors' perimeter and the lack of closed flooring inside the cabinet, which is necessary for power and control cables entering. 


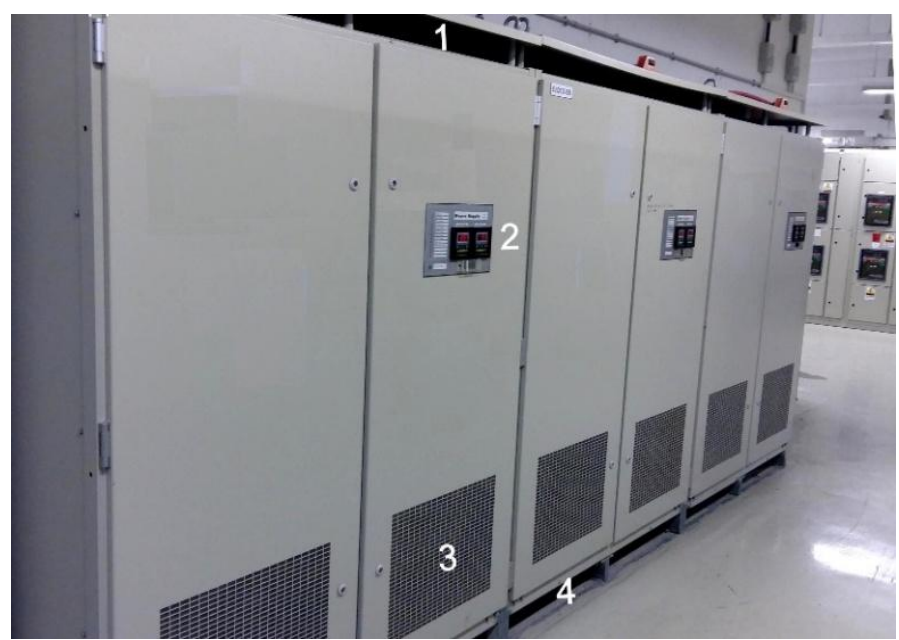

Fig1. Design of power plant's BC. The figures indicate the "weaknesses" of cabinets that allow unobstructed access of HEMP inside the cabinet.

All these weaknesses allow unobstructed access of electromagnetic emission (including HEMP) into the internal part of the BC cabinets. Thus, the measures intended to improve survivability of DCAPS should first deal with improvement of the cabinets' design.

The ventilation grid 2 should be substituted by a special panel of honeycomb structure, based on "waveguides-below-cutoff" (Fig. 2) consisting of a set of small diameter metal pipes with the pipe's length to diameter ratio of $4: 1$.

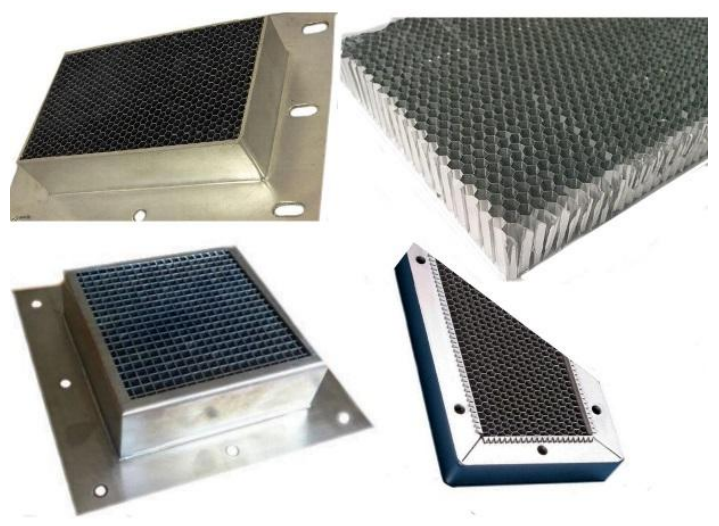

Fig2. Honeycomb ventilation panels protecting from electromagnetic emission

A ventilation panel such as this will allow the air flow in, but will not allow the electromagnetic emission inside the cabinet. These panels should cover other parts of the cabinet which are open for ventilation purposes (1 and 4$)$.

A metal frame, projecting height wise above the screen and keys of the unit 2, should be welded around the open panel of the programming and measuring unit (2), located on the external surface of the cabinet's door. This frame should be equipped with a steel 3-4 mm thick or aluminum (4-6 mm) small door. However, steel would be preferable. A seal designed as a conductive rubber cord should be glued to the door's edges contacting the frame.
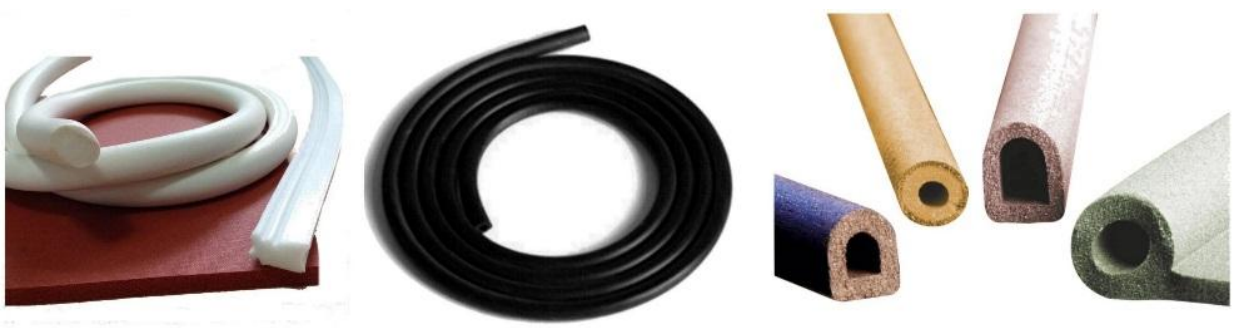

Fig3. Seals made of a conductive rubber cord to be installed on HEMPs protected cabinet doors 
All other seals made of non-conductive rubber running along the perimeter of each cabinet's door should be replaced by the above mentioned seals (made of conductive rubber).

The floor surface, free of cables, should be covered with a conductive metalized fabric (Fig. 4) laid on a supporting metal grid.
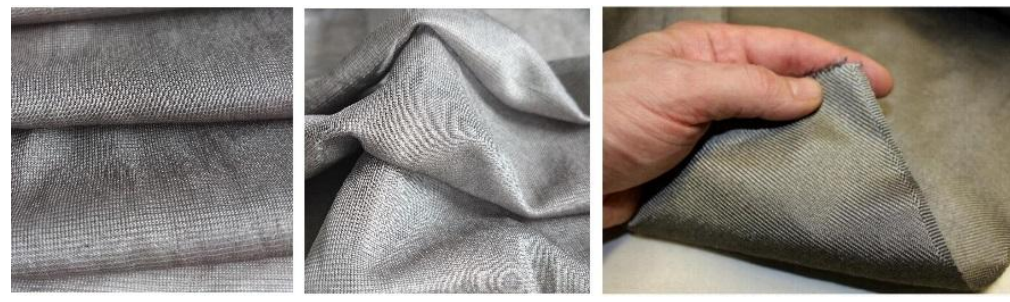

Fig4. Conductive metalized fabric to cover open parts of the cabinet's floor

\section{IMPROVEMENT OF DCAPS'S ELECTRICAL CIRCUIT}

In order to improve DCAPS's resilience, some other elements were incorporated into it (Fig. 5), i.e.:

- varistor blocks with high energy dissipating capacity R1 - R7, installed on AC and DC current busbars;

- varistor blocks with medium energy dissipating capacity R8 - R23, installed in BC cabinets and accumulator batteries' terminals to limit the voltage pulse amplitude applied to DCAPS equipment;

- chokes L1 - L8 that limit the amplitude of current pulse, which can flow in DCAPS equipment circuits under HEMP impact;

- special elements of the grounding system L9 - L10, R24-R25, K1 - K2.

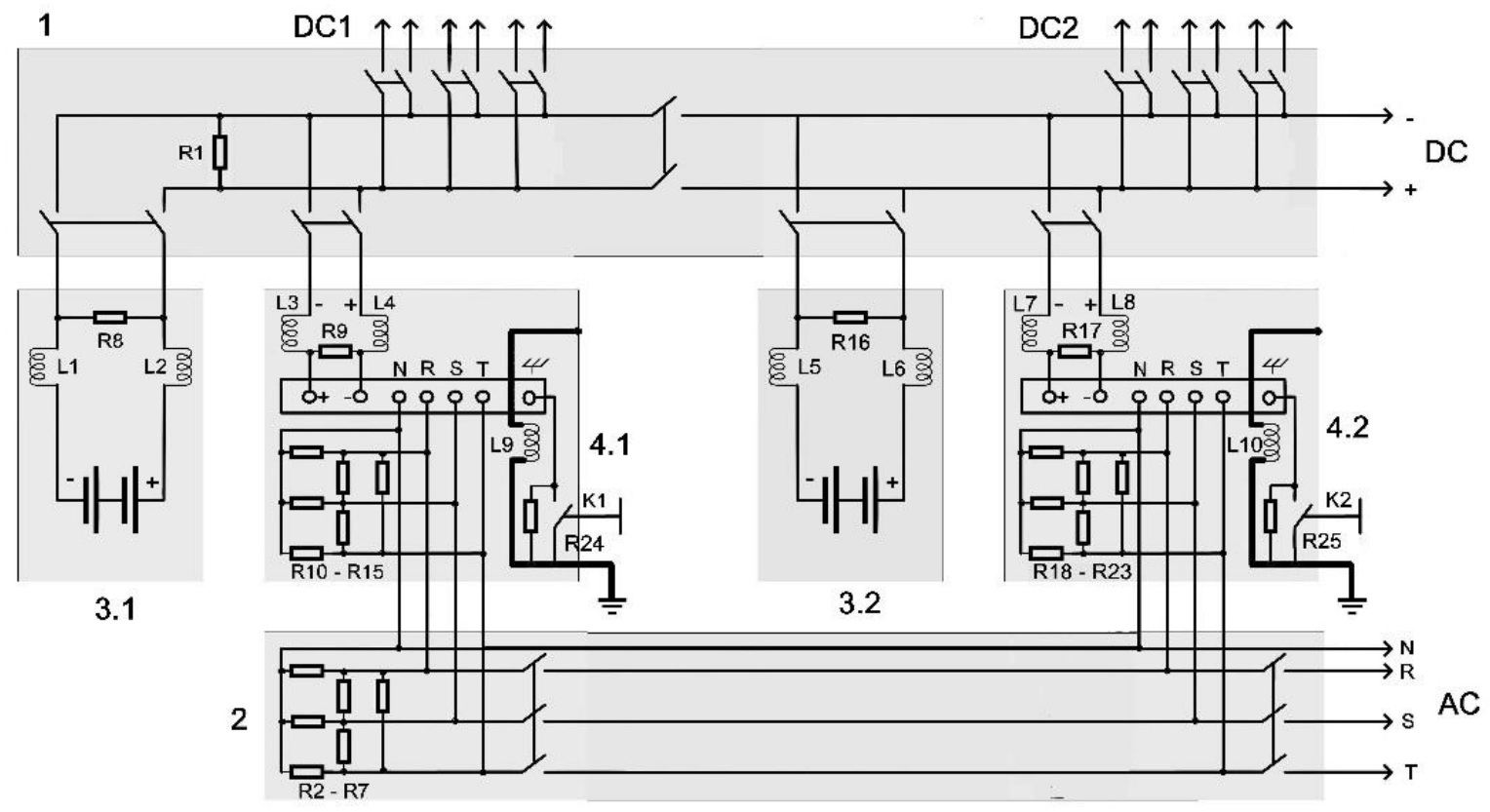

Fig5. General electric circuit diagram of HEMP protected DCAPS. $1-D C$ switchboard (DC1, DC2); $2-A C$ switchboard; 3 -accumulator batteries; 4 -BC

\section{Protection Elements For DCAPS}

It is recommended to use high energy varistor blocs manufactured by EPCOS, Semicode, Dean Technology, Inc., Dongguan Uchi Electronics Co., etc., built in standard enclosures with maximum dimensions of $135 \mathrm{~mm} \times 118 \mathrm{~mm} \times 27 \mathrm{~mm}$, see Fig. 6. Such varistor blocks s are capable of single current pulses of $8 / 20 \mathrm{~ms}$ with amplitude up to $100 \mathrm{kA}$ and dissipating energy of $2 \mathrm{~kJ}-3 \mathrm{~kJ}$, or even up to $10 \mathrm{~kJ}$. Pulse response time is $15 \mathrm{~ns}-25 \mathrm{~ns}$, according to the manufacturer's data (generally, real time response is more). Varistors of this type should be outsized (by nominal voltage) as they are designed to ensure protection against HEMP high-voltage pulse, rather than against surge transient 
overvoltage normally presented in the system. For example, for DCAPS with nominal voltage of $250 \mathrm{VDC}$, such varistors can have 420VDC activation voltage (residual voltage or clamping voltage is $840 \mathrm{~V})$.
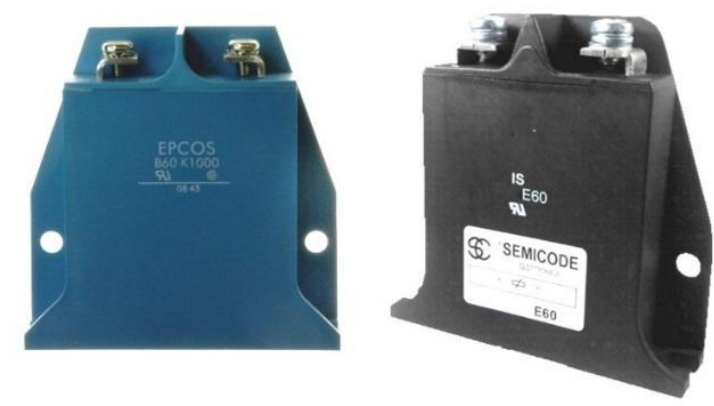

Fig6. Varistor blocks with high dissipated energy and pulse currents up to $100 \mathrm{kA}$

Upon HEMP impact, a residual voltage of $840 \mathrm{~V}$ does not damage the wire and power cables insulation, as they are designed to withstand much higher pulse voltages of several kilovolts. On the other hand, such a big safety factor ensures reliable and damage-free varistor operation within the long period.

As medium dissipated energy varistors, it is also recommended to use varistor blocks made by the above mentioned manufacturers or Littlefuse Company, see Fig. 7. Such varistors, designed for installation inside the $\mathrm{BC}$ cabinet or directly on the battery block buses, are tolerable to single pulse currents up to $30 \mathrm{kA}-40 \mathrm{kA}$, have lower dissipated pulse energy (300J - 500J), smaller maximum size (usually, $60 \mathrm{~mm} \times 55 \mathrm{~mm} \times 14 \mathrm{~mm}$ ), and lower redundant operating voltage, e.g. 320VDC (clamping voltage is $650 \mathrm{~V}$ ).
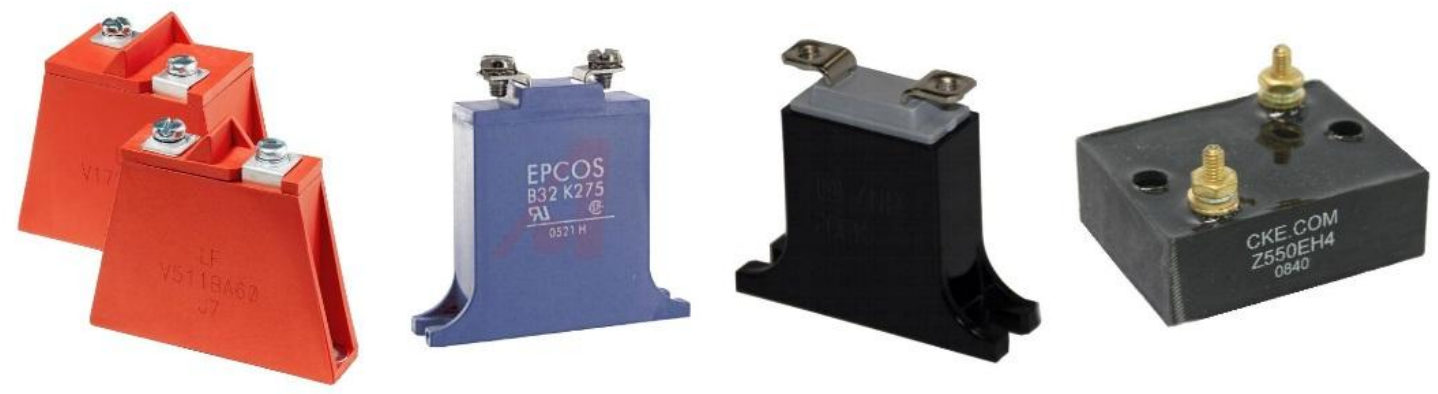

Fig7. Varistor Blocks with medium energy dissipating capacity and pulse currents up to $40 \mathrm{kA}$.

High-power chokes L1 - L8 suitable for use in DCAPS as HEMP filters are manufactured by the American company CWS, see Fig. 8, Table 1.

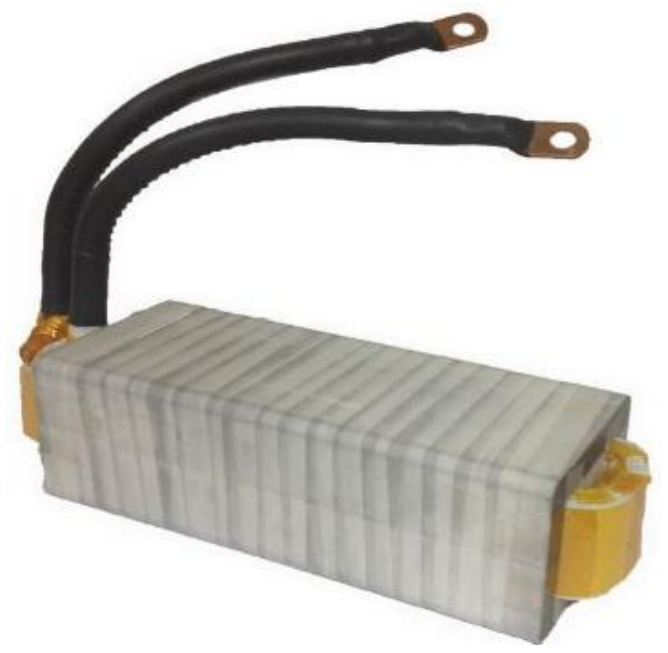

Fig8. High power cored HCS series choke for currents up to 1,000 A manufactured by CWS Company under special technology using special material

International Journal of Research Studies in Electrical and Electronics Engineering (IJRSEEE) Page | 29 
Improvement of Survivability of a Power Plant's Direct Current Auxiliary Power System under Electromagnetic Pulse (HEMP)

Table1. Key parameters of powerful HCS Series chokes with a core

\begin{tabular}{|c|c|c|c|c|c|c|}
\hline Choke Type & Max. & Inductance at & \multicolumn{2}{|c|}{ Resistance (Impedance) } & \multirow{2}{*}{$\begin{array}{c}\text { Dimensions, } \\
\text { Cum }\end{array}$} \\
\cline { 5 - 6 } & $\begin{array}{c}\text { Continuous } \\
\text { Current, } \mathbf{A}\end{array}$ & $\mathbf{3 0 0 A}, \boldsymbol{\mu H}$ & $\begin{array}{c}\mathbf{D C}, \\
\mathbf{m} \boldsymbol{\Omega}\end{array}$ & $\begin{array}{c}\mathbf{1} \\
\mathbf{M H z}, \\
\mathbf{k} \boldsymbol{\Omega}\end{array}$ & $\begin{array}{c}\mathbf{G H z}, \\
\mathbf{M} \boldsymbol{\Omega}\end{array}$ & \\
\hline HCS-631M-450A & 450 & 249 & 4.5 & 1.56 & 1.56 & $393 \times 118 \times 97$ \\
\hline HCS-301M-1000A & 1000 & 244 & 4.5 & 1.53 & 1.53 & $431 \times 228 \times 101$ \\
\hline
\end{tabular}

The chokes are connected into the cut of power DC cables exiting the BC through the open part of the $\mathrm{BC}$ flooring. When arranging the chokes and varistor blocks in the BC cabinets, they should be located on the cabinet's floor so that they are covered with a conductive fabric separating the protected area of the cabinet from external space. It is advisable to arrange a special metal compartment on the BC floor to accommodate the chokes and varistor blocks. Cables "free" from induced HEMP should enter the inner part of the cabinet.

Control cables of alarm and signal circuits that enter and exit the BC cabinets shall be protected by detachable ferrite filters, Fig. 9. These filters should be located directly at the point of the cables' entry into the cabinet.

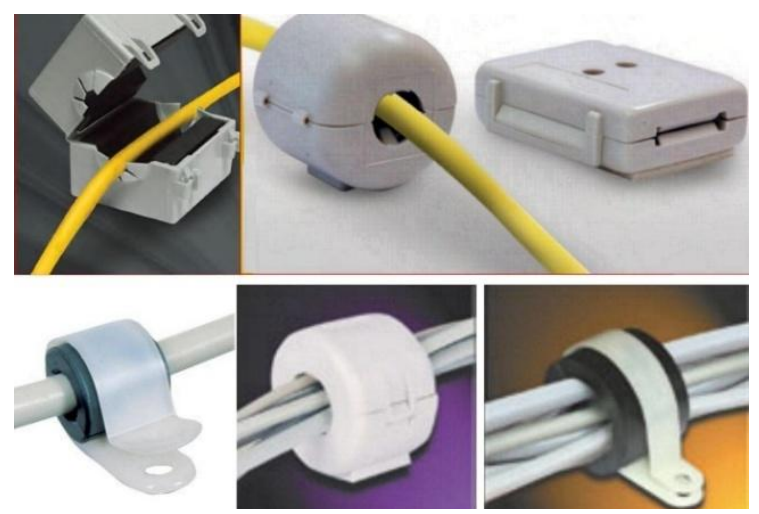

Fig9. Ferrite HEMP filters installed on control cables without cutting them.

The features of ferrite filters, as well as recommendation on their use, are provided in [3].

Components of cabinet's grounding system and internal "ground" of BC: L9 - L10, R24-R25, K1 $\mathrm{K} 2$ are intended to realize a so called "special floating ground", the detailed description of which is provided in [4].

\section{SPARE MODUleS}

In order to improve survivability of DCAPS, it is very important to ensure availability of spare elements, modules or spare BC (as shown in Fig. 1) in addition to the above mentioned measures and technical facilities. Moreover, spare BC should have a well-protected (using the above mentioned equipment) cabinet and should be completely disconnected from all external circuits and external cables. The truth, however, is that it is rather expensive to have a powerful spare $\mathrm{BC}$ on each unit of a power plant. Storing of spare electronic elements and electronic blocks (printed circuit boards) of BC in a special closed container will be more affordable for mass use. Additionally, the printed circuit boards should be placed into conductive plastic packages (Fig. 10), whereas the packages should be placed into aluminum containers with a close fitting cover (Fig. 11). The containers, with spare electronic components, should be stored close to the $\mathrm{BC}$, rather than in a remote warehouse.
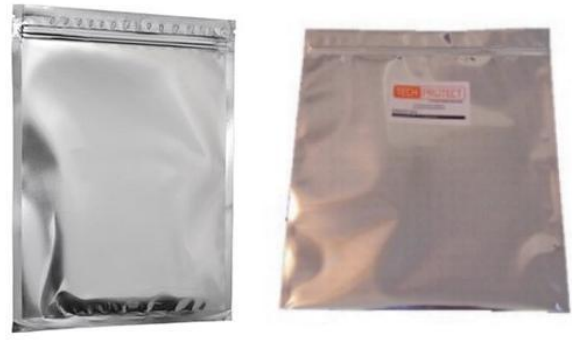

Fig10. Protective conductive plastic packages to store spare printed circuit boards of BC. 

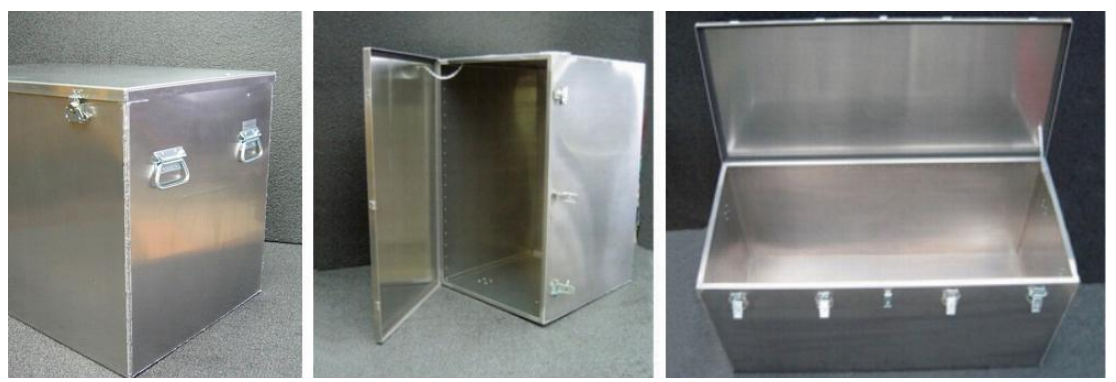

Fig11. Protective aluminum containers to store spare electronic elements and modules of BC.

\section{Conclusions}

The simple measures and affordable technical facilities described in the article result in significant improvement of survivability of a very important power plant's system - DCAPS under HEMP impact, and reduce the risk of complete "loss" of a block or even a power plant in the case of impact.

\section{REFERENCES}

[1] Gurevich, V., Protection of Substation Critical Equipment Against Intentional Electromagnetic Threats. Wiley, London, 2017, $240 \mathrm{p}$.

[2] Gurevich, V.,Cyber and Electromagnetic Threats in Modern Relay Protection. - Taylor \& Francis Group, Boca Raton, 2015, 205 p.

[3] Gurevich, V., The problem of correct choice of ferrite beads, Electrical Engineering \& Electromechanics, 2016, No. 2, pp. 71-73.

[4] Gurevich, V., The Issues of Electronic Equipment Grounding at the Power Facilities, International Journal of Research Studies in Electrical and Electronics Engineering (IJRSEEE), 2017, vol. 3, issue 1, pp. 11- 19.

\section{AUTHOR'S BIOGRAPHY}

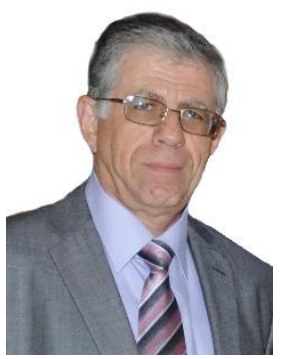

Vladimir I. Gurevich, was born in Kharkov, Ukraine, in 1956. He received an M.S.E.E. degree (1978) at the Kharkov Technical University, named after P. Vasilenko, and a Ph.D. degree (1986) at Kharkov National Polytechnic University.

His employment experience includes: teacher, assistant professor and associate professor at Kharkov Technical University, and chief engineer and director of Inventor, Ltd.

In 1994, he arrived in Israel and works today at Israel Electric Corp. as a Senior specialist and Head of section of the Central Electric Laboratory. He is the author of more than 200 professional papers and 15 books and holder of nearly 120 patents in the field of electrical engineering and power electronics. In 2006 he was Honorable Professor with the Kharkov Technical University.

Citation: Vladimir Gurevich (2017). Improvement of Survivability of a Power Plant's Direct Current Auxiliary Power System under Electromagnetic Pulse (HEMP), International Journal of Research Studies in Electrical and Electronics Engineering (IJRSEEE), 3(2), pp.26-31, DOI: http://dx.doi.org/10.20431/24549436.0302004.

Copyright: (C) 2017 Vladimir Gurevich. This is an open-access article distributed under the terms of the Creative Commons Attribution License, which permits unrestricted use, distribution, and reproduction in any medium, provided the original author and source are credited 